\title{
Submicron focusing of XUV radiation from a laser plasma source using a multilayer Laue lens
}

\author{
M. Reese · B. Schäfer · P. Großmann • A. Bayer • \\ K. Mann · T. Liese · H.U. Krebs
}

Received: 8 October 2010 / Accepted: 18 November 2010 / Published online: 10 December 2010

(C) The Author(s) 2010. This article is published with open access at Springerlink.com

\begin{abstract}
The focusing properties of a one-dimensional multilayer Laue lens (MLL) were investigated using monochromatic soft X-ray radiation from a table-top, laserproduced plasma source. The MLL was fabricated by a focused ion beam (FIB) structuring of pulsed laser deposited $\mathrm{ZrO}_{2} / \mathrm{Ti}$ multilayers. This novel method offers the potential to overcome limitations encountered in electron lithographic processes. Utilizing this multilayer Laue lens, a line focus of XUV radiation from a laser-induced plasma in a nitrogen gas puff target could be generated. The evaluated focal length is close to the designed value of $220 \mu \mathrm{m}$ for the measurement wavelength of $2.88 \mathrm{~nm}$. Divergence angle and beam waist diameter are measured by a moving knife edge and a far-field experiment, determining all relevant secondorder moments based beam parameters. The waist diameter has been found to be approximately $370 \mathrm{~nm}$ (FWHM).
\end{abstract}

\section{Introduction}

The spectral range of the "water window" (wavelength of 2.3-4.4 nm) offers a highly interesting regime for studying water dissolved samples, since they are reasonably transparent for the applied radiation, whereas carbon shows a significantly higher absorption. This is of particular interest for

M. Reese $(\bowtie) \cdot$ B. Schäfer · P. Großmann · A. Bayer · K. Mann Laser-Laboratorium Göttingen e.V., Hans-Adolf-Krebs-Weg 1, 37077 Göttingen, Germany

e-mail: michael.reese@1lg-ev.de

Fax: +49-551-503599

T. Liese $\cdot$ H.U. Krebs

Institut für Materialphysik, Universität Göttingen,

Friedrich-Hund-Platz 1, 37077 Göttingen, Germany high resolution microscopy in biology, life sciences, polymer studies, and many other fields of research [1]. In this regime, the refractive index $n$ is close to unity and effective refractive optics are not available. Diffractive optical elements such as Fresnel zone plates have been developed and are successfully employed for both focusing and imaging of soft X-ray radiation since many years [1, 2]. Zone plates are annular gratings of alternating transparent and nontransparent (or phase shifting) materials with a narrowing grating constant, whose diffractive orders coincide at a common point [1]. Zone plates for soft X-ray or XUV radiation are usually manufactured by electron lithography and reactive ion etching [3-5]. With the help of these techniques, outermost zone widths of $12 \mathrm{~nm}$ have been demonstrated [5], determining the achievable resolution limit. A further decrease in zone width, however, turns out to be more and more challenging with standard methods.

To achieve even finer structures and higher resolution, while maintaining the required high aspect ratio, an alternative approach is the use of multilayers of high and low absorbing (or phase-shifting) material, satisfying the Fresnel zone plate law. These structures are known as multilayer Laue lenses (MLLs), which have been demonstrated in oneor two-dimensional geometry [6-8] for hard X-ray radiation, creating a line or a point focus. However, as to our knowledge, they have not yet been applied as diffractive optics in the soft X-ray regime so far. Krebs et al. have recently shown that MLLs can be effectively manufactured by pulsed laser deposition (PLD) and focused ion beam (FIB) techniques $[9,10]$. These novel structures show nearly no cumulative roughness and no damage from rough dicing or polishing techniques.

In this paper, we report on the integration of a onedimensional multilayer Laue lens manufactured by PLD and FIB into a measurement setup based on a table-top laser- 
driven plasma source $[11,12]$. Using spectrally purified line emission from a Nitrogen plasma $(\lambda=2.8787 \mathrm{~nm})$, the optical properties of this focusing element were characterized in two different experiments. First, the beam caustic around the waist was monitored using a moving knife-edge method in the near focal region; thereafter, a movable CCD camera was used to examine the far-field distribution behind the MLL.

The presented data demonstrate the feasibility of submicron focusing with MLLs in the soft X-ray spectral range. The development of improved diffractive optical elements in combination with table-top soft X-ray sources can accomplish interesting experiments such as microscopy for a large community of users.

\section{Experimental}

\subsection{Fabrication of the multilayer Laue lens}

A one-dimensional Multilayer Laue Lens for the soft X-ray regime has recently been fabricated from laser deposited $\mathrm{ZrO}_{2} /$ Ti multilayers (see PLD standard setup in [13]) using a focused ion beam setup (FEI Nova Nanolab), featuring an outermost zone width of $35 \mathrm{~nm}$, which determines the minimal theoretically achievable line focus of about $42 \mathrm{~nm}$, and a

Table 1 Design parameters of the multilayer Laue lens

\begin{tabular}{ll}
\hline Number of zones & 85 \\
Outermost zone width & $34.6 \mathrm{~nm}$ \\
Focal length for $2.88 \mathrm{~nm}$ & $220 \mu \mathrm{m}$ \\
Aperture size of one multilayer package & $3.67 \times 15 \mu \mathrm{m}^{2}$ \\
Size of the center beam stop & $11 \times 15 \mu \mathrm{m}^{2}$ \\
\hline
\end{tabular}

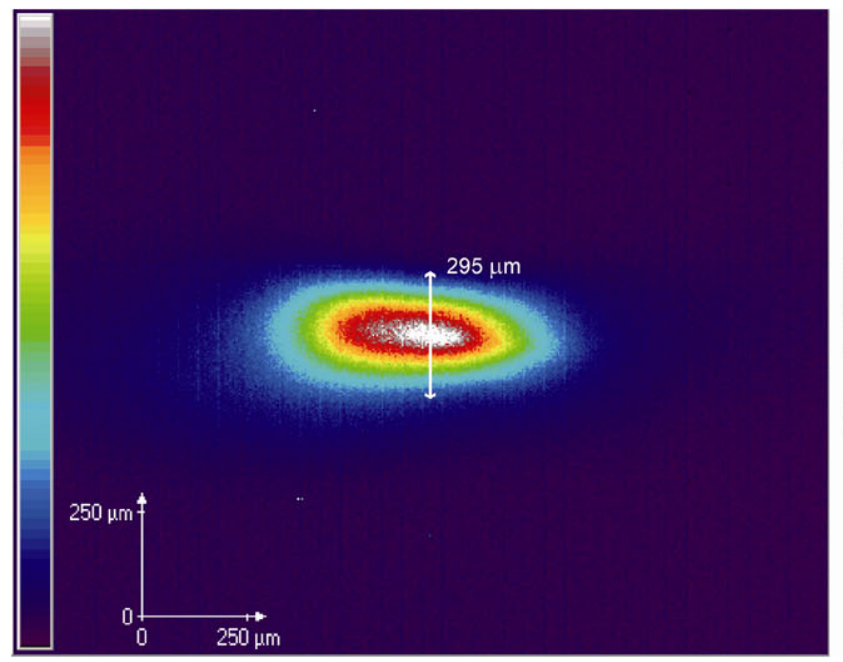

focal length of approximately $220 \mu \mathrm{m}$ for the wavelength of $2.88 \mathrm{~nm}$. Details of the complex deposition and assembling processes are described in $[9,10]$.

The design parameters for plane wave illumination are summarized in Table 1. They apply for ideal planar wave illumination. An SEM picture of the fabricated MLL consisting of two multilayer packages is displayed as insert of Fig. 2.

\subsection{Characterization of the multilayer Laue lens with XUV radiation}

For characterization of the described MLL a table-top laserdriven plasma source for generation of soft X-rays developed at Laser-Laboratorium Göttingen is employed [11, 12]. A Nd:YAG laser (InnoLas Spitlight $600 @ 1064$ nm, 800 mJ, $6 \mathrm{~ns}, 10 \mathrm{~Hz}$ ) is focused into a pulsed gas jet injected into the vacuum chamber by a Proch-Trickl valve [14], leading to the formation of a hot and dense plasma which in turn emits characteristic soft X-ray radiation. By selecting the target gas and suitable blocking filters, the emission characteristics of the plasma can be varied from broadband to singleline emission. For monochromatic emission within the water window, Nitrogen was chosen as target material in combination with a titanium filter to deliver a single emission line at a wavelength of $2.8787 \mathrm{~nm}$. A pinhole camera image of the nitrogen plasma is displayed in Fig. 1 (left), indicating a plasma size of $295 \mu \mathrm{m} \times 823 \mu \mathrm{m}\left(1 / e^{2}\right)$ (right). The lens was aligned to focus the short axis of the plasma. Thus, the effective plasma width is $295 \mu \mathrm{m}$.

This XUV source is part of the measurement setup sketched in Fig. 2. An experimental chamber containing the MLL is attached to the source chamber by a vacuum tube. Due to the relatively large distance $D$ of $830 \mathrm{~mm}$ from the

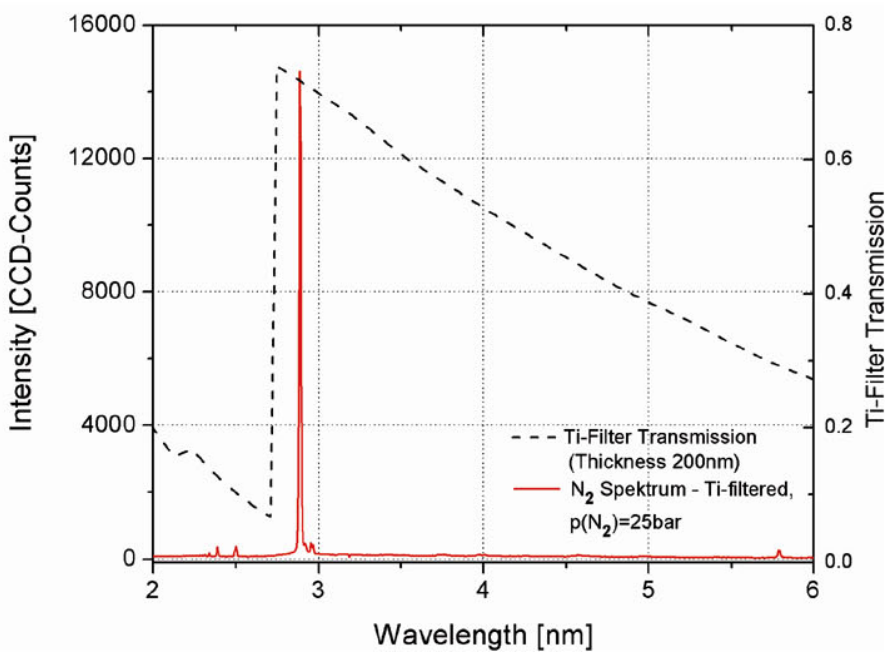

Fig. 1 Left: Pinhole camera image of the nitrogen plasma indicating a size of $295 \mu \mathrm{m} \times 823 \mu \mathrm{m}\left(1 / e^{2}\right)$. Right: XUV emission spectrum filtered by $200 \mathrm{~nm}$ thick titanium foil; only the $1 s^{2}-1 s 2 p$ emission line $(\lambda=2.8787 \mathrm{~nm})$ is transmitted 


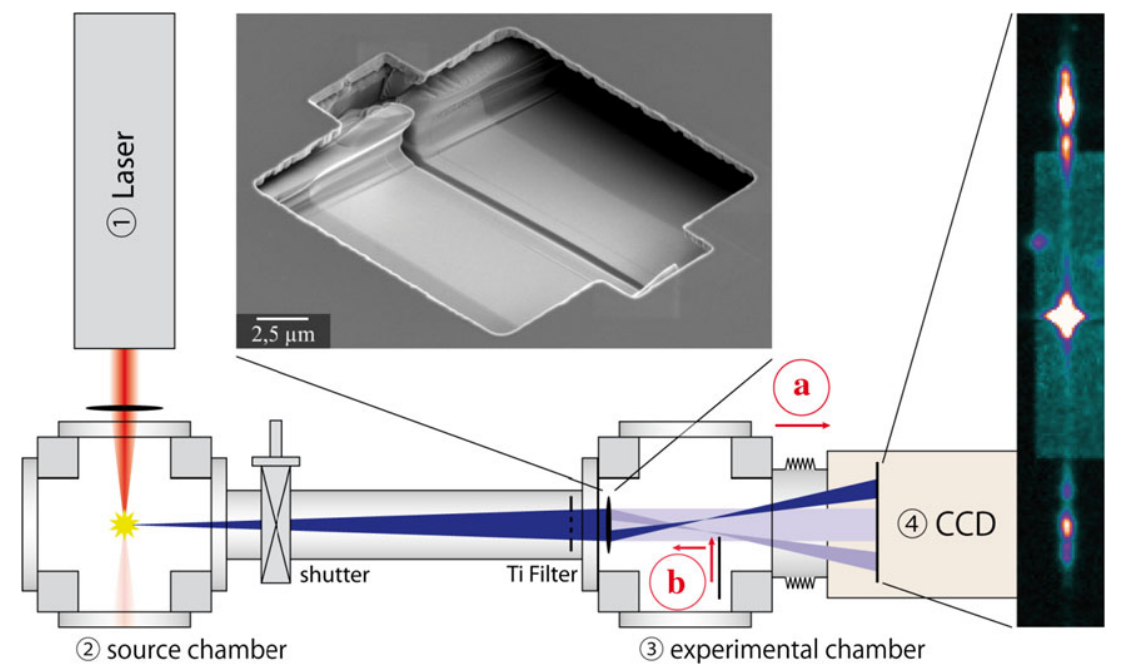

Fig. 2 Schematic drawing of the experimental setup. A Nd:YAG laser (1) is used as energy source for XUV plasma generation in a pulsed gas jet (2). Monochromatic radiation from the plasma at $2.88 \mathrm{~nm}$ wave length illuminates the multilayer Laue lens positioned in the experimental chamber (3) as a nearly planar wave. A back-side illuminated CCD camera (4) used as detector can be translated along the beam di-

source, the illumination wave-front on the MLL lens can be assumed as approximately planar. A back-thinned backside illuminated CCD camera (Roper Scientific PI-SX: 1k, $1024^{2}$ Pixels, $13 \times 13 \mu \mathrm{m}^{2}$ ) is mounted on a translation stage behind the MLL and can be moved along the optical axis $z$. Furthermore, a system of shadowing apertures, a titanium filter and a gold covered supporting aperture ensure that the lens is illuminated by XUV radiation only, and no indirect light from the source can reach the camera.

A typical CCD camera image recorded with the described setup is displayed in Fig. 2 (right), indicating the +1 st diffraction orders of both lenses as well as the bright undiffracted 0th order. The soft X-ray diffraction signal can be monitored with sufficient intensity by integration over 1200 Pulses at $10 \mathrm{~Hz}$. Obviously, the intensity profiles of the 1st diffraction orders show a distinct structure; this aberration can be attributed to imperfections of the MLL. Furthermore, background XUV light transmitted through the $\mathrm{Au}(500 \mathrm{~nm})$ covered $\mathrm{Si}_{3} \mathrm{~N}_{4}$ membrane $(100 \mathrm{~nm})$ is also registered (rectangular structure).

To investigate the focusing properties of the MLL two experiments were carried out. At first, since the pixel size of the CCD prevents the direct investigation of the beam waist, a direct measurement of the generated focal line was performed by recording the beam caustic in a moving knifeedge (MKE) experiment. For this purpose a metal wire (10 $\mu \mathrm{m}$ diameter) was mounted onto two piezo-driven linear motors (Mechonics PS30, $50 \mathrm{~nm}$ step width) with closed loop positioning control, translating it perpendicular and along the optical axis. Beam widths at different positions rection to determine the divergence angle of the propagating beam (a); a wire is used as moving knife edge to obscure the beam at defined positions using piezo-driven motors (b). The camera image shown on the right was recorded at a distance of approximately $5 \mathrm{~mm}$ from the MLL displaying the bright 0 th diffraction order and the weaker +1 st diffraction orders of the two multilayer packages

around the waist are determined from the integral intensity decrease monitored with the camera. Alignment of the knife edge parallel to the focal line was accomplished by monitoring the shadow of the wire on the camera illuminated by transmitted background light.

In addition, the propagating beam was also monitored by acquiring intensity profiles at various distances between the CCD camera and the MLL. Due to alignment imperfections of the MLL, only the beam of one multilayer package has been considered for the characterization measurements described below.

\section{Results}

\subsection{Moving knife edge experiment}

Figure 3 displays data from moving knife edge experiments for several positions of the blade along the beam close to its waist. With the moving knife edge moving gradually into the beam, it is incrementally obscured. Therefore, the curves represent the decrease of the integrated intensity with the moving knife edge location, thus resembling the integral over the beam profile. According to ISO 11146:1999(E) [15], the beam width is evaluated and the results are given in FWHM with the approximation of a near-Gaussian intensity distribution. As an example, the data for the smallest recorded beam diameter with the fitted error function as well as the corresponding Gaussian profile is plotted in Fig. 4. This smallest measured beam diameter is determined to $374 \mathrm{~nm}$ at $365 \mu \mathrm{m}$ relative moving knife edge position. 

integrated soft X-ray intensity registered by the CCD detector against the relative moving knife edge position. The different curves represent several experiments along the optical axis
Fig. 3 Normalized plot of the

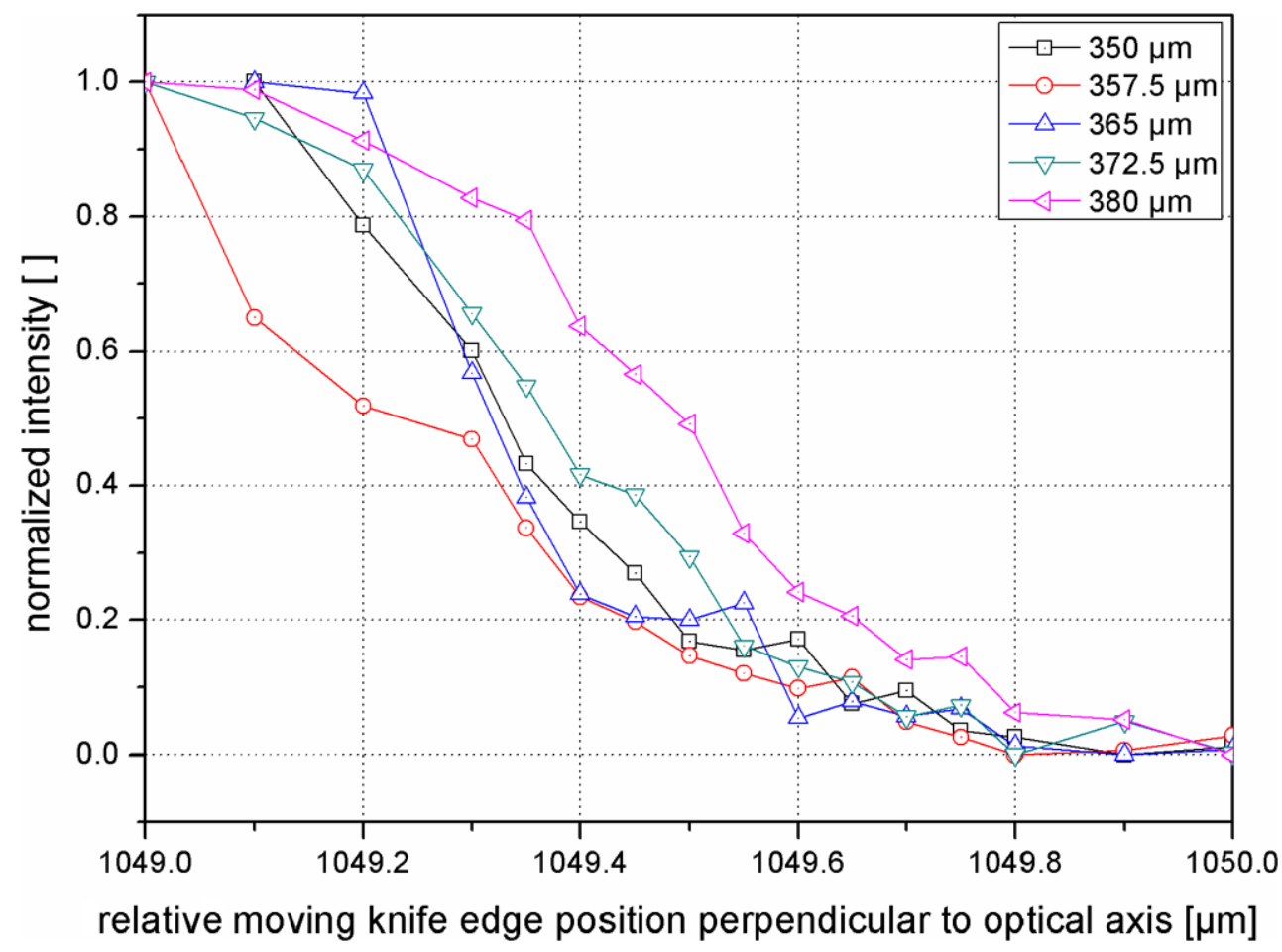

Fig. 4 Plot of the integrated soft X-ray intensity registered by the CCD detector against the relative knife edge positions, representing the measurement closest to the beam waist. The error function corresponding to the parameters gained in the second moment evaluation is plotted into the data and its derivate is shown, representing the approximate Gaussian beam profile

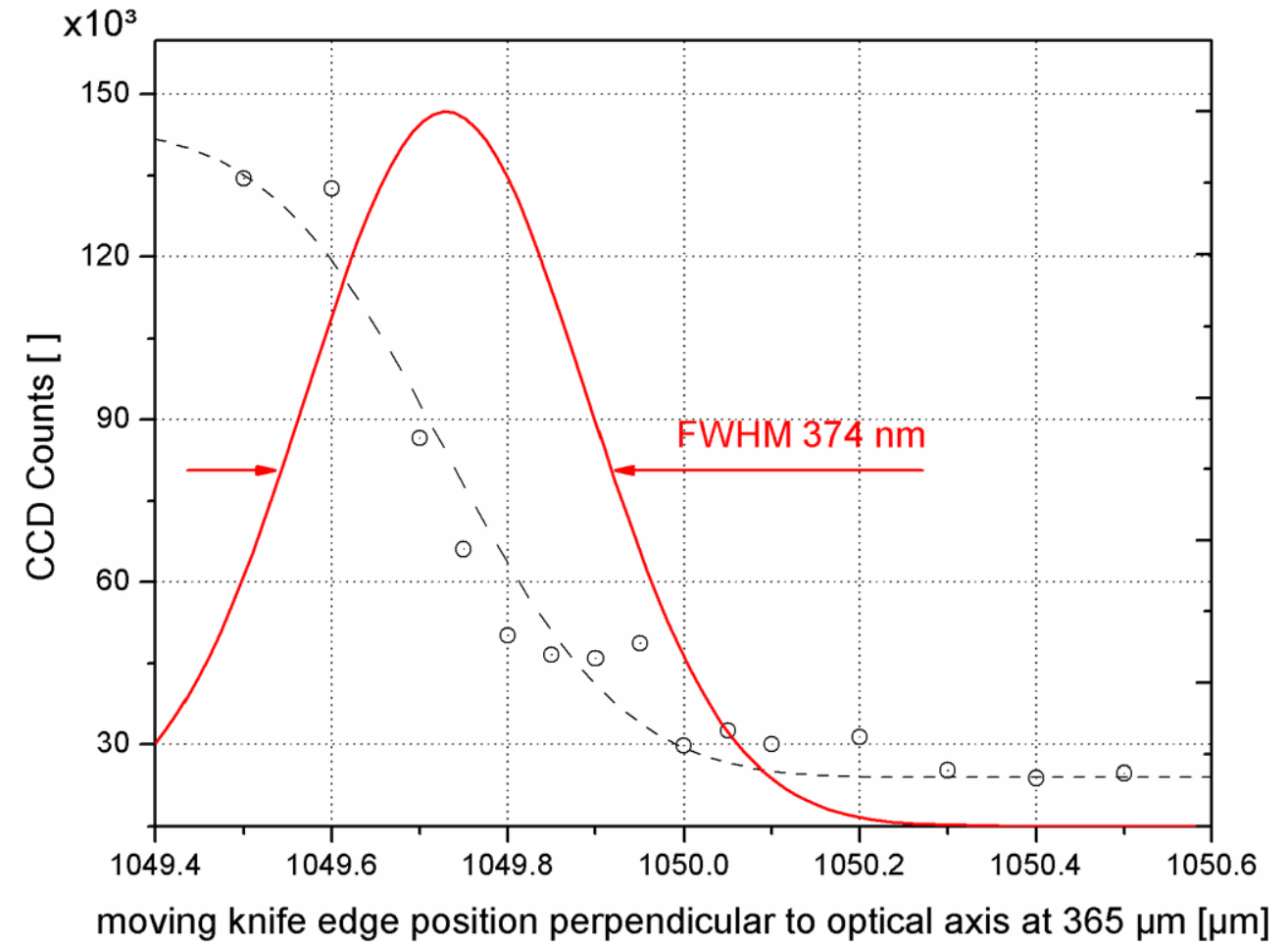

In Fig. 5, the determined beam widths are plotted against their relative position along the optical axis. A hyperbolic fit indicates the beam profile near the beam waist shows a Gaussian behavior. From this fit, the smallest beam width of $375 \pm 30 \mathrm{~nm}$ at a relative moving knife edge position of $367 \pm 2 \mu \mathrm{m}$ is found.

\subsection{Beam divergence experiment}

In order to independently investigate the propagation characteristics of the radiation behind the MLL, beam profiles were recorded at various detector distances ranging from approximately 5 to $85 \mathrm{~mm}$, as displayed in Fig. 6. After 
Fig. 5 Caustic of the determined beam widths against the relative position of the knife edge along the principle optical axis, including a hyperbolic fit to the data
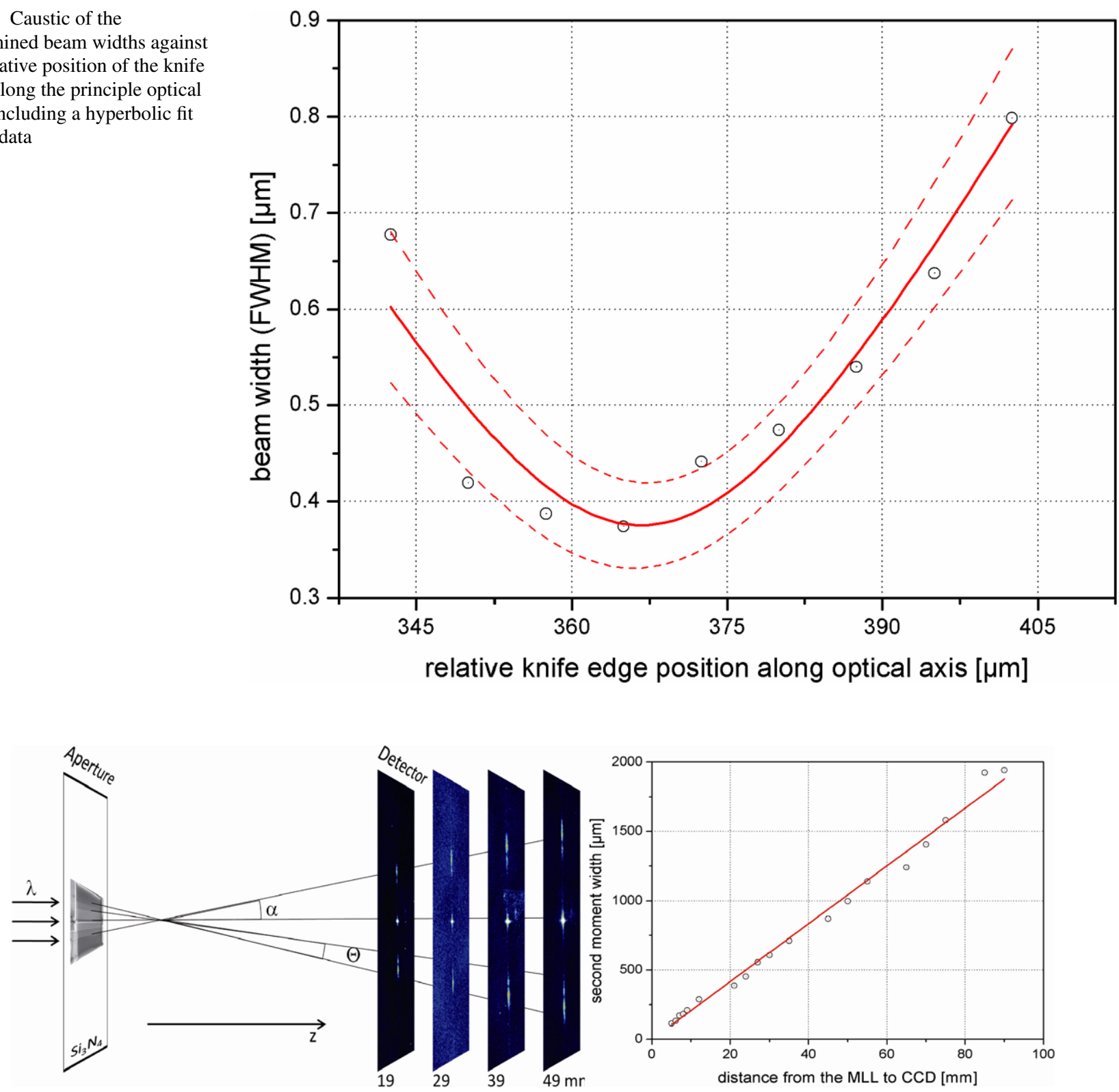

Fig. 6 CCD camera images taken at various distances behind the MLL for an illumination wavelength of $2.8787 \mathrm{~nm}$, displaying 0th and +1 st diffraction orders. $\alpha$ describes the angle between the 0th and 1 st orders, $\Theta$ the divergence angle of the +1 st order diffracted beam itself. The graph shows the second moment beam widths of the +1 st

background subtraction, the first and second image moments of the first diffraction orders were calculated [16] to evaluate the second moment beam widths according to ISO 11146:1999(E) [15]. A linear regression of the calculated second moment beam widths of the +1 st diffraction orders plotted against the distance $z$ of the detector from the MLL yields the corresponding beam divergence angle $\Theta=20.4$ mrad. For comparison, beam broadening due to diffraction at the aperture of the MLL is more than an order of magnitude smaller than the measured divergence. order diffracted beam plotted against the distance of the camera from the MLL, yielding the divergence angle from a linear regression. The approximate absolute position of the focal spot was determined from the extrapolated crossing point of the 0 th and 1 st order beams

\subsection{Evaluation of propagation characteristics}

From the hyperbolic fit to the beam waist, all relevant second-order moment based beam parameters can be determined according to ISO 11146:1999(E) [15]. However, the far-field divergence angle is determined more precisely from the beam divergence experiment; hence, the value of Sect. 3.2 is used for the subsequent evaluation of the focal length of the MLL. The beam width $d(z)$ along the caustic 
Table 2 Properties of the multilayer Laue lens

\begin{tabular}{ll}
\hline Divergence angle & $\begin{array}{l}20.4 \pm 0.3 \mathrm{mrad} \\
\text { (divergence angle experiment) }\end{array}$ \\
Beam waist diameter (FWHM) & $\begin{array}{l}375 \pm 30 \mathrm{~nm} \\
\text { (moving knife edge experiment) }\end{array}$ \\
Rayleigh length & $31.3 \pm 2.5 \mu \mathrm{m}$ \\
Focal length & $205 \pm 24 \mu \mathrm{m}$ \\
\hline
\end{tabular}

is given by

$d(z)=\sqrt{d_{0}^{2}+(z \cdot \Theta)^{2}}$.

Taking into account that $d(z=f)$ is equal to the second moment width of the MLL aperture size of $4.24 \mu \mathrm{m}$, a focal length $f=205 \mu \mathrm{m}$ can be calculated. This value computed from the combined data of the two experiments is in good agreement with the design value of the lens $(f=220 \mu \mathrm{m})$. Moreover, a simple geometrical evaluation of $f$ from the angle $\alpha$ between 0 th and 1 st diffraction order yields a similar result $(f=206 \mu \mathrm{m})$. Table 2 summarizes all results of the evaluation.

From the fitted data of the knife edge measurement, the beam waist diameter is determined considerably larger than the designed value. Alignment imperfections and vibrations of the experiment are a possible source of error leading to larger measured beam widths in the moving knife edge experiment. They are expected to be in the region of roughly $100 \mathrm{~nm}$ each. Thus, the result for the beam width can be interpreted as an upper limit. Other sources of error are the plasma source size and diffraction from the MLL aperture. However, their contributions were identified as insignificant.

On the other hand, the large beam waist can be explained by fabrication related aberrations of the lens, especially irregularly deposited multilayers, which have been identified by SEM inspection. The moving knife edge experiment confirms this hypothesis since a distinct fine structure of the intensity distribution is not only registered in the detector plane, but also near the beam waist. Quantifying these aberrations, a beam propagation factor $M^{2} \sim 6$ is calculated from the fit of the experimentally determined beam caustic, indicating a considerable decrease of beam quality by lens aberration.

\section{Conclusions}

In summary, we have shown that submicron focusing of XUV radiation from a laser plasma source can be reached using a MLL in Laue diffraction geometry. For this, a MLL fabricated by PLD and FIB was integrated in a laboratory scale laser plasma-based XUV source and characterized with monochromatic radiation for a wavelength of $2.88 \mathrm{~nm}$. Two experiments determining the beam propagation behind the optical element show that its focal length is at about $220 \mu \mathrm{m}$ and the measured beam waist diameter is in the order of $370 \mathrm{~nm}$ (FWHM). For this high value, fabrication induced aberrations of the lens as well as experimental error sources could be responsible. Further improvement of the fabrication process to get high quality structures is on the way.

Acknowledgement This work was supported by the Deutsche Forschungsgemeinschaft (SFB 755 "Nanoscale Photonic Imaging”).

Open Access This article is distributed under the terms of the Creative Commons Attribution Noncommercial License which permits any noncommercial use, distribution, and reproduction in any medium, provided the original author(s) and source are credited.

\section{References}

1. D. Attwood, Soft X-rays and Extreme Ultraviolet Radiation (Cambridge University Press, Cambridge, 1999)

2. J. Thieme, G. Schmahl, D. Rudolph, E. Umbach, X-ray Microscopy and Spectromicroscopy (Springer, Berlin, 1998)

3. G. Schmahl, D. Rudolph, B. Niemann, AIP Conf. Proc. 75, 225 (1981)

4. C. David, B. Kaulich, R. Medenwaldt, M. Hettwer, N. Fay, M. Diehl, J. Thieme, G. Schmahl, J. Vac. Sci. Technol. B 13, 2762 (1995)

5. W. Chao, J. Kim, S. Rekawa, P. Fischer, E.H. Anderson, Opt. Express 17, 17699 (2009)

6. N. Kamijo, Y. Suzuki, H. Takano, S. Tamura, M. Yasumoto, A. Takeuchi, M. Awaji, Rev. Sci. Instrum. 74, 5101 (2003)

7. S. Tamura, M. Yasumoto, N. Kamijo, K. Uesugi, A. Takeuchi, Y. Terada, Y. Suzuki, J. Phys. Conf. Ser. 186, 012075 (2009)

8. H.C. Kang, G.B. Stephenson, C. Liu, R. Conley, R. Khachatryan, M. Wieczorek, A.T. Macrander, H. Yan, J. Maser, J. Hiller, R. Koritala, Rev. Sci. Instrum. 78, 046103 (2007)

9. T. Liese, V. Radisch, H.-U. Krebs, Rev. Sci. Instrum. 81, 073710 (2010)

10. T. Liese, V. Radisch, I. Knorr, M. Reese, P. Großmann, K. Mann, H.U. Krebs, doi:10.1016/j.apsusc.2010.10.076

11. S. Kranzusch, C. Peth, K. Mann, Rev. Sci. Instrum. 74(2), 969 (2003)

12. C. Peth, F. Barkusky, K. Mann, J. Phys. D, Appl. Phys. 41, 105202 (2008)

13. H.U. Krebs, O. Bremert, Appl. Phys. Lett. 62, 2341 (1993)

14. D. Proch, T. Trickl, Rev. Sci. Instrum. 60, 713 (1989)

15. ISO/FDIS 11146:1999(E), Lasers and laser-related equipmenttest methods for laser beam parameters-beam widths, divergence angles and beam propagation factor (1999)

16. M.D. Abramoff, P.J. Magelhaes, S.J. Ram, Biophotonics Int. 11(7), 36-42 (2004) 